\title{
Region of interest extraction using redundant wavelet transform and unsupervised techniques on thermal imaging
}

\author{
B. Ortiz-Jaramillo*, J. Garcia-Álvarez*, J. Rodríguez-Sotelo*, D. Peluffo-Ordóñez* and G. Castellanos-Domínguez* \\ *G. Control y Procesamiento Digital de Señales, Universidad Nacional de Colombia, Manizales, bortizj@unal.edu.co
}

Most applications in the analysis of thermal images can detect changes in patterns [1]. Thus, thermal patterns provide sufficient information of the structure for a particular device, giving the opportunity to alert and perform some preventive action in the process.

The wavelet transform is useful to characterize this kind of change, because the localized of the scale information gives the nature of the representation. Thereafter, relevant details are preserved when scale is changed in the representation space, excluding other details that not provide information about regions of interest [2]. On the other hand, the patterns obtained in the characterization phase can be clustered by using unsupervised techniques. Those patterns are measured with an adequate metric to determine the principal cluster of the dataset. Then, additional criteria can be employed to initialize the centroids of the cluster to obtain a better outcome. This criteria is supported by no homogeneous representation of the cluster, justified by the nature of the thermal distribution: its distribution has smooth changes in the image, making difficult the automatic detection of those regions. In this way, a change in the representation space is necessary to make easy the clustering process. Thus, changes in the initial groups can be detected, when the cluster is initially identified in normal condition of the devices and the image representation space is wavelet. A pattern change is defined as the change in the number of clusters or in the size of some cluster. It represents a fault candidate in the device, making possible to take decision about the actual state of the device.

State of the art proposes several metrics that can be used like Minkowski metric, Mahalanobis distance, correlation, among others [3]. In this context another parameters can be introduced to improve the results of the technique: initialization methods, e.g., max-min, and automatic estimation of the number of clusters, e.g., spectral techniques [4]. In this way, clustering algorithm includes the centroid initialization that ensures local maximum, and the optimal selection of the number of clusters, giving a better approximation of the region of interest [5]. Our proposed method is tested over a CFRP006 plate of $30 x 30 \mathrm{~cm}$ and 25 defects but the frame used for the results only shows 17 defects with different size and depth. In this way other metrics are proposed to evaluate the performance of the segmentation method, e.g., Jaccard metric is a measure of the correlation between the real and estimate segmentation.

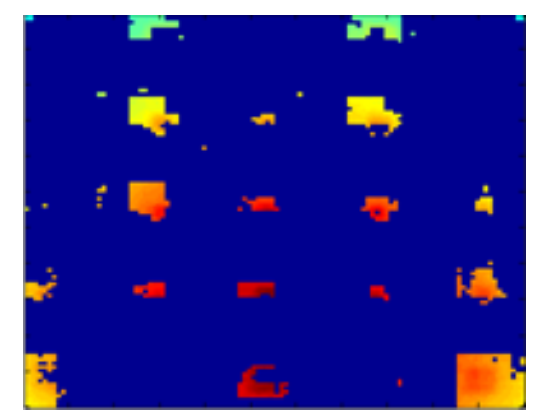

Fig. 1. CFRP006 plate segmented using proposed method

Figure 1 shows the segmentation over the previously described plate using the proposed method and the k-means clustering algorithm.

Assigned Class

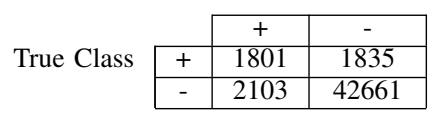

TABLE I

CONFUSION MATRIX FOR PROPOSED METHOD 
Table I presents the confusion matrix for the image shown in Figure. From the Table we can obtain the true positives rate $(T P R=0.4953)$, the false positives rate $(F P R=0.0470)$, the distance to perfect segmentation $\left(d=\sqrt{T F P^{2}+(1-T V P)^{2}}=\right.$ $0.5069)$ and the Jaccard metric ( $\mathrm{JC}=$ number of true positive/(number of true positive + number of false positives + number of false negatives $)=0.3138$ ). The qualitative results show that the more important area of the region of interest is selected by the initial cluster algorithm.

\section{CONCLUSiOnS}

An automatic region of interest extraction algorithm based in the wavelet representation space is presented to extract the appropriate characteristics of thermal image, thus, the labeling of those characteristics is possible with a clustering algorithm. The proposed method shows good qualitative results and comparative results of confusion matrix. Moreover this method offers the possibility of editing of the following: the number of cluster criteria, the appropriate metric to calculate the cluster. Some algorithms that take advantage of the Gaussian nature of thermal heating as the Bayesian, Parzen, among others, can be implemented in this method for optimization.

\section{REFERENCES}

[1] H. Benitez-Restrepo, A. Bendada, C. Ibarra-Castañedo, and H. Loaiza. Analytical forward and inversion modeling of infrared phase images using thermal quadrupole theory in pulsed phase thermography. In Advanced Infrared Technology and Applications, León - México, 2007.

[2] B. Zhen Zhanga, M. Siliang, H. Liua, and Y. Gonga. An edge detection approach based on directional wavelet transform. Computers \& Mathematics with Applications, 57(8):1265 - 1271, April 2009.

[3] V. Perlibakas. Distance measures for pca-based face recognition. Pattern Recognition Letters, 25(16):711-724, April 2004.

[4] Yu Stella X. and Shi Jianbo. Multiclass spectral clustering. In ICCV '03: Proceedings of the Ninth IEEE International Conference on Computer Vision, page 313, Washington, DC, USA, 2003. IEEE Computer Society.

[5] J. L. Rodríguez Sotelo, D. Peluffo, D. Cuesta Frau, D. Peluffo Ordónez, and G. Castellanos Domínguez. Non-parametric density-based clustering for cardiac arrhythmia analysis. Computers in cardiology. CINC, 2009. 